\title{
A study of fluid flow characteristics using micro structured surfaces produced by WEDM
}

\author{
Mohammed A.H. Al-fahham, Samuel Bigot, Agustin Valera Medina \\ Cardiff School of Engineering, Cardiff University, Cardiff, UK, e-mail: bigots@cf.ac.uk
}

\begin{abstract}
This work presents an approach where biomimetic structures are tested for stabilisation and reduction of the boundary layer to reduce flashback propensity. Micro structures have been designed to have the shape of microriblets similar to those found in shark skin and other vegetable organisms. Various texture geometries were produced by micro Wire Electro Discharge Machining. These geometries were tested in a bespoke flame channel under isothermal conditions to observe the flow boundary profile with the aim of using combustion as a next step to determine the resistance to flashback propagation. CFD analyses were also performed using in-house simulation software Hydro3D utilising LES modelling. Experimental and simulation results were compared and showed an improvement of the boundary layer using the micro-riblets based on their structural patterns.
\end{abstract}

Keywords: Boundary layer, Biomimetic, flashback, micro WEDM, immersed boundary method.

\section{Introduction}

Drag reduction is the resistive force fluid shows on a body in either closed or open channel flows (laminar or turbulent). Laminar flows look smooth and orderly whereas turbulent flows look chaotic and random. Drag generally is higher in turbulent flows. Turbulence has exceptional importance in all studies involving fluid engineering such as aircraft design, submarines, ships, pipelines, etc. Many techniques are used to reduce drag based on the application. For example, suction of the boundary layer over air plane wings or cooling the surface to slow the energy dissipation. Many technical approaches are inspired from nature. Living nature provokes the engineers to mime the unique solutions that some animals and plants are using to get food or to protect themselves. An observation of a large variety of surface structures on the skin of the fast sharks was done by Bechert et a [1]. He inspected three species of sharks and modelled skin samples from different locations to identify the most important skin structure in terms of swimming speed. He suggested that the tiny ribs are the important features. Bechert et al. [2] did extensive research studying the size of riblets, optimal cross sectional shapes and ratio between riblet spacing and riblet height. He reported a $10 \%$ reduction with an optimal shape. The angle between the flow direction and aligned riblets (Yaw angle) has been studied by Bechert et al. [3]. They reported the values of angles that fix or increase drag reduction. Koeltzsch et al. [4] investigated the velocity field over convergent and divergent riblet patterns. They concluded that close to the wall their convergent and divergent riblets have considerable differences with time-averaged streamwise velocity and streamwise velocity fluctuations. Bixler and Bhushan [5] studied four microstructured surfaces inspired on rice leaves and fabricated with photolithography. They reported a drag reduction of around $12 \%$. Such results are very promising for other applications where drag is a performance detrimental.
However researchers agree that one of the biggest challenges is the accurate and cost effective production of surfaces with micro structures having the desired shapes and required rigidity. Bechert et al. [3] reported that the major experimental effort consisted in the manufacturing of suitable surfaces. In their work they used a combination of printing and casting procedure to obtain a $1.5 \mathrm{~mm}$ long and $0.5 \mathrm{~mm}$ spaced ribs in aluminum. Koeltzsch et al. [4] coated an experimental pipe with a special riblet film to achieve smaller patterns. The same coating technique was used by Dean and Bhushan [6] in a rectangular duct. A channel wall was covered with a shark skin like texture used by Jin and Herwig [7] to study drag reduction in terms of enthalpy change. Replica Molding, a technique to duplicate shape, size, and patterns of features on a master mold was also used by Chen et al. [8]. Bixler and Bhushan [5] used a Silicon master pattern to have micro -sized feature surfaces.

However, Stainless steel has not been widely used so far to produce micro-structures for such applications, while it would be an ideal material due to its wide availability and its mechanical and thermal properties. The reason for this is mainly due to the difficulties in finding adequate machine tools able to achieve the required micro grooves or structures. One promising technology that could achieve this is micro Electrical Discharge Machining ( $\mu$ EDM). It refers to a process with sub $\mu \mathrm{m}$ material removal units, performed using an electrode containing micro scale geometries, i.e. dimensions from 5 to $500 \mu \mathrm{m}$. Compared to other technologies, its main advantage is the ability to machine high aspect ratio micro features on any conductive material with good surface quality, i.e. average roughness $\mathrm{Ra}$ down to $200 \mathrm{~nm}$ or below, irrespective of hardness. Machining is performed by a sequence of electrical discharges occurring in an electrically insulated gap between a tool electrode and a workpiece. During the discharge pulses, a high temperature plasma channel is formed in the gap, causing evaporation and melting of the workpiece. Debris is removed by the resulting explosion pressure, enabling machining. 


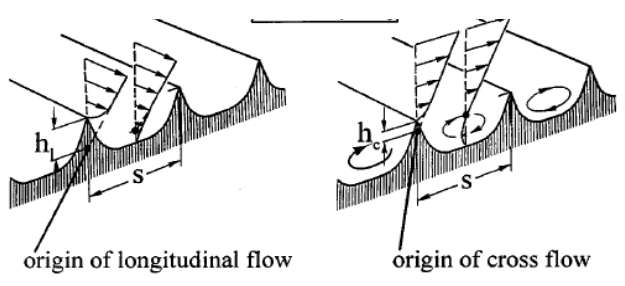

Fig. 1. Longitudinal and cross flow on a ribbed surface [2]

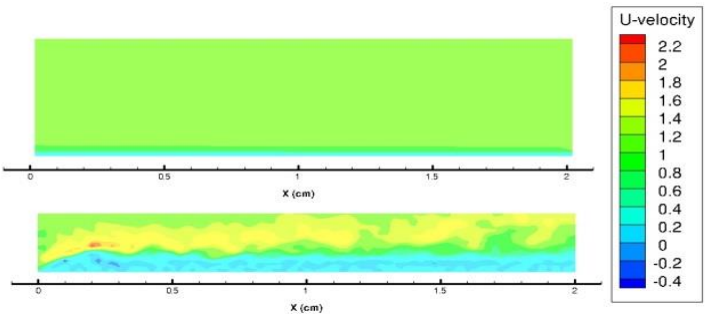

Fig. 2. Micro-structured surface effects on flow patterns. Up) With riblets; Down) Without riblets.

$\mu$ EDM has been the focus of many research activities in last decays [9]. In this paper the applicability of micro Wire Electrical Discharge Machining ( $\mu \mathrm{WEDM})$ for the production of complex micro riblets on a stainless steel surface is assessed. In particular, three new surface patterns have been designed as part of a project aiming at improving the flow in gas turbine combustors to reduce flashback when using highly hydrogenated fuel blends. The obtained surfaces are then used to study their influence on fluid flow characteristics using both simulation and experimental data.

\section{Theoretical background and Simulation}

For any moving viscous fluid over a surface there will be friction which is caused by the allure between fluid molecules and the surface of an object in the fluid, also caused by intermolecular attraction within the bulk fluid. This kind of bond causes fluid drag which is the deleterious transfer of energy between the surface and fluid flow. This drag depends on the nature of flow either laminar or turbulent. A laminar flow occurs with low velocities whilst a turbulent flow is perceived in most real life applications with an increase of velocity. This transition can be described by the Reynolds number $(\mathrm{Re})$, which represents the ratio of internal forces to viscous forces,

$$
R e=\frac{U L}{v}
$$

Where $U$ is the stream velocity, $L$ the characteristic length and $v$ the kinematic viscosity.

For most engineering application the fluid flow is turbulent which means high drag forces. Therefore, the microstructured riblets would help reduce the anisotropic flow and control vortices on the skin of the system, which are naturally present in turbulent flows. The riblets hold and presumably pin any vortices generated in the viscous layer, which cause a drag reduction as shown in Fig. 1. Thus making riblets an appropriate component in many applications.

\section{Methodology}

\subsection{CFD Analyses}

The numerical analysis for turbulent flows is a complicated issue that needs a hard work and enormous computational resources to simulate or predict the flow behaviour under turbulent conditions. Even with existing supercomputers the cost are still sometimes prohibitive.

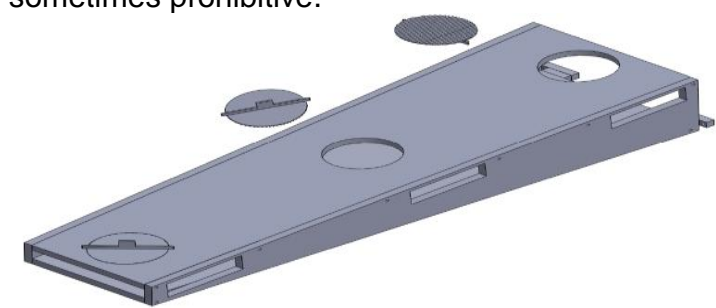

Fig. 3. Test rig: micro textured inserts and windows.

Thus, an immersed boundary method was used to simulate the presence of the micro-structured surfaces in the fluid flow stream in this work. The software Hydro3D was employed for the task. One of the advantages of using an immersed boundary method is that the mesh (grid) generation is much easier and that it reduces computational efforts, especially with the micro dimensions that need a huge amount of computing resources. This issue comes from the basics that the mesh in a flow domain is generated as a Cartesian grid and the flow equation is solved at these grids, thus representing any solid domain (Immersed body) by equivalent forces. The effect of such forces is added to the nearest points in the flow domain. Such techniques reduce the computing time and sources required for the numerical calculations.

Fig. 2 shows the simulation result of fluid flow over a flat plate with and without the presence of riblets. This numerical simulation was initially used to test many riblet shapes (convex/concave riblets, saw shape, triangle shape and diamond shapes) and assess which one demonstrate the best performance while being producible with our available manufacturing resources.

\subsection{Experimental rig}

A variable cross section channel was constructed to test the flow over the micro structured surfaces. The channel is $1.5 \mathrm{~cm} \times 10 \mathrm{~cm}$ cross sectional inlet and 80 $\mathrm{cm}$ length with an adjustable bottom part to have different outlet aspect ratios. Each side wall has three quartz windows $2.4 \mathrm{~cm} \times 9.6 \mathrm{~cm}$ to give a better access to view the flow development and effects of the micro structure surface on the flow as shown in Fig. 3. These windows are flashed accurately with the inner side of the wall to avoid any flow disturbance. Hotwire anemometry was used to obtain velocity fluctuations.

As shown in Fig. 3, the stainless steel circular inserts containing the micro textures to be studied can be integrated in front of each window. $\varnothing 80 \mathrm{~mm}$ inserts were initially considered following the WEDM machine height limitation, which was used to produce the features. Initial tests demonstrated non negligible inaccuracies linked to the difficulty in keeping appropriate micro-wire alignments with such a height and due to the cost and time required to produce micro features on large areas it was decided to use $\varnothing 25 \mathrm{~mm}$ stainless steel inserts instead.

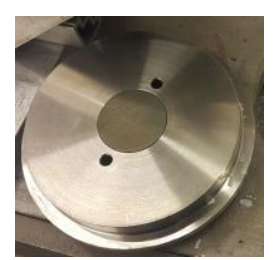




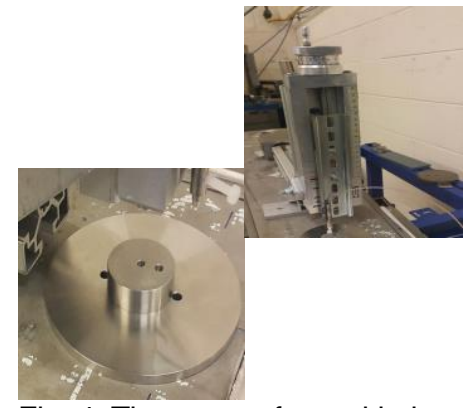

Fig. 4. The test surface with the measuring equipment For correlation purposes, boundary layer thickness and drag reduction were used. Hotwire anemometry (HWA) was used to study the velocity behaviour and turbulent intensity in the normal direction to the wall at two positions, i.e. $5 \mathrm{~mm}$ before and after the structure, Fig 4. For each position (in X-direction), the boundary layer was measured using velocity data provided by HWA. To do so, the Hotwire was incrementally displaced away from the surface until the ratio between the velocity measured by the hotwire and the main stream velocity reaches 0.99 , which should represent the thickness of the boundary layer.

Trials were repeated 3 times. Measuring steps in the normal direction were firstly very fine from the wall to $300 \mu \mathrm{m}$ from the wall, i.e. $20 \mu \mathrm{m}$ steps. Then the step was increased to $50 \mu \mathrm{m}$ until $1 \mathrm{~mm}$ from the wall. Finally, $100 \mu \mathrm{m}$ steps were used until $2.2 \mathrm{~mm}$ from the wall where in all cases the mean velocity was equal to 0.99 of the mean stream velocity. A Re 70,000 was evaluated and therefore also used in the simulations. A Dantec MiniCTA Hot Wire Anemometry system was used, having a $1.2 \%$ measurement error.

\subsection{Texture Manufacture and Design}

\subsubsection{Manufacturing Strategy}

A $\varnothing 100 \mu \mathrm{m}$ wire was used as cutting tool, and due to the discharge gap the smallest achievable feature identified was $130 \mu \mathrm{m}$. A small gap is generally linked to a smaller discharge energy and consequently to smaller removal of material and longer machining times, making it almost impossible to cut through the bulk of a workpiece.

Thus, for each produced structure, the inserts top surface was first machined "flat" with the highest energy to efficiently align the top surfaces to the wire axis, a process which requires the removal of a significant amount of material. Six other "flat" cuts were then performed with gradually smaller energy and smaller discharge gaps to achieve a better surface finish. A final cut following the required profiles was then performed with the smallest energy with a targeted roughness around $200 \mathrm{~nm} \mathrm{Ra}$, assuming this would ensure that the flow would be affected mainly by the tested geometries and not by the roughness. An average surface roughness of $200 \mathrm{~nm}$ Ra measured using a White light interferometer.

\subsection{Micro textures designs}

The aim of the initial design was to test the smallest feature achievable using the design shown in Fig. 1, namely $130 \mu \mathrm{m}$ wide riblets. A depth of $65 \mu \mathrm{m}$ was set to maintain an optimal depth-width ratio of 0.5 [2]. Fig. 5 shows the resulting structure. For the other structures, the aim was to try and replicate the lotus leaf and shark skin textures, with the limitations of the
WEDM process, to produce truly 3D structures rather than a $2-1 / 2 D$ structure. The key reasoning was that drag effects are 3-dimensional and therefore the textures should provide turbulence reduction in both the streamwise and cross flow directions by being positioned at a suitable angle to avoid energy losses. To achieve this, multiple wire passes were performed after rotating the circular stainless inserts thus providing some 3 dimensional complexities to the textures.

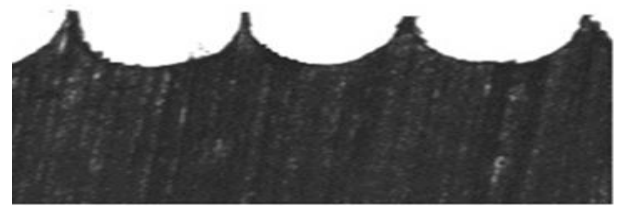

Fig. 5. First Riblet Design

Figure 6 shows the key designs that were produced; one inspired by lotus and two by shark skin: one called diamond design and the more complex one called shark skin design. The lotus design aimed to imitate the fine pins of lotus leaves with 2 close perpendicular wire cuts, while the diamond design and shark skin designs imitating shark skin denticles with 2 wire cuts at $60^{\circ}$ to each other. The shark skin design used an additionally smaller cut at a $30^{\circ}$ angle in an attempt to model smaller riblets that appear on the animal denticles.

For the lotus design, the grooves width was kept at $130 \mu \mathrm{m}$ with a depth of $65 \mu \mathrm{m}$. Due to the need of deeper grooves for the diamond and shark skin textures, the grooves were set at $260 \mu \mathrm{m}$ width with $130 \mu \mathrm{m}$ depth. The spacing between grooves was set to $25 \mu \mathrm{m}$ for the lotus design and to $280 \mu \mathrm{m}$ for the other two.

Figures 7 and 8 show the actual machining results for the diamond and the shark designs. The geometrical results obtained with the shark skin design deviated significantly from the targeted features, mainly due to alignments issue as shown in Fig. 8. Details of the resulting geometries for the lotus and the diamond design are given in Table 1.

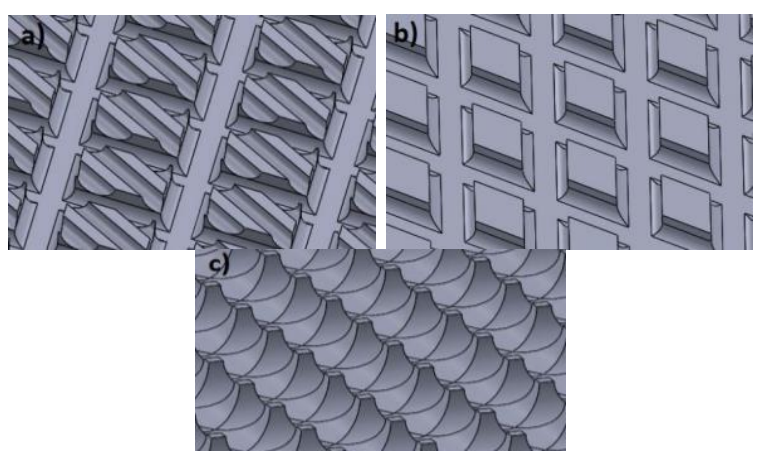

Fig. 6. a) Shark skin, b) Diamond, c) Lotus

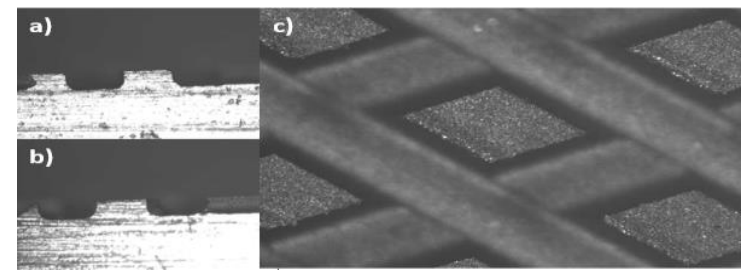


Fig. 7. Produced Diamond Design

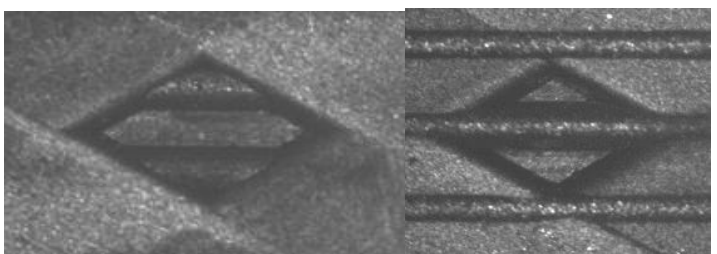

Fig. 8. Produced Shark Skin Design

Table 1

Manufactured structures

\begin{tabular}{|c|c|c|c|}
\hline \multicolumn{2}{|c|}{ Diamond Design Features } & Target & Measured \\
\hline \multirow{3}{*}{$\begin{array}{l}\vec{J} \\
0 \\
0 \\
\infty\end{array}$} & Width of groove $(\mu \mathrm{m})$ & 260 & 306.2 \\
\hline & Depth of groove $(\mu \mathrm{m})$ & 130 & 144.6 \\
\hline & Width of riblet $(\mu \mathrm{m})$ & 280 & 247.9 \\
\hline \multirow{4}{*}{ 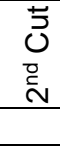 } & Width of groove $(\mu \mathrm{m})$ & 260 & 312.2 \\
\hline & Depth of groove $(\mu \mathrm{m})$ & 130 & 124.5 \\
\hline & Width of riblet $(\mu \mathrm{m})$ & 280 & 241.9 \\
\hline & Angle between cuts $\left(^{\circ}\right)$ & 60 & 61 \\
\hline \multicolumn{2}{|c|}{ Lotus Design Features } & Target & Measured \\
\hline \multirow{3}{*}{ 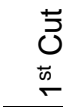 } & Width of groove $(\mu \mathrm{m})$ & 130 & 143.6 \\
\hline & Depth of groove $(\mu \mathrm{m})$ & 65 & 49.3 \\
\hline & Width of riblet $(\mu \mathrm{m})$ & 25 & 10.7 \\
\hline \multirow{4}{*}{ 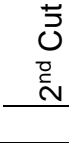 } & Width of groove $(\mu \mathrm{m})$ & 130 & 151.4 \\
\hline & Depth of groove $(\mu \mathrm{m})$ & 65 & 65.8 \\
\hline & Width of riblet $(\mu \mathrm{m})$ & 25 & 7.3 \\
\hline & Angle between cuts $\left(^{\circ}\right)$ & 90 & 86 \\
\hline
\end{tabular}

\section{Results and discussion}

Due to unforeseen complexity in the preparation of the data files, at this stage, numerical simulation were not performed for the lotus structure. For other structures, the numerical results shown in Table 2 suggest a reduction in boundary layer thickness and thus drag reduction. To validate the simulation results, three experimental tests have been performed so far, for the initial smooth surface, for the lotus and for the diamond structures.

Other numerical and experimental tests are currently being performed and will be available soon.

These limited set of results suggest good agreement between the numerical simulation and the experiments. However, as only one small set of measurements has been performed so far, further tests are required to validate the accuracy and repeatability of the proposed experimental method. In addition, different orientations of the structures, in accordance to the fluid flow, still need to be tested and may lead to the identification of optimum orientations.

In any case, numerical results showed that the velocity in-between the riblets, which normally lay in the laminar sublayer, is more stable and with low turbulence (Fig. 2) when using micro structures, thus leading to wall friction reduction and drag reduction. In addition, structured geometries can be optimised to obtained better flow pattern as shown in the velocity fields given in Fig. 9.

Table 2

Resulting information and Boundary layer

Design $\quad$ Boundary Layer Thickness $(\mathrm{mm})$

Numerical Experimental

\begin{tabular}{lcc}
\hline Smooth surface & 2 & 1.98 \\
Lotus & & 1.9 \\
Diamond & 1.9 & 1.93 \\
Concave Riblets & 1.92 & \\
Convex Riblets & 1.98 & \\
\hline
\end{tabular}

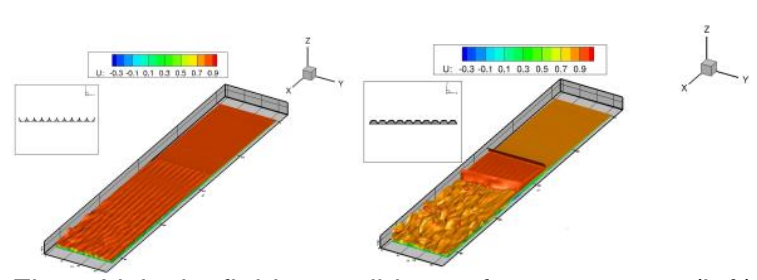

Fig 9. Velocity field over riblet surfaces concave (left) and convex (right)

\section{Conclusions}

The numerical results show good agreement with flow experiments, showing that the CFD approach can be expanded even further to more complex geometries for further studies. Expected experimental results put more light on the actual behaviour of fluids through riblets and give more options and ideas to develop both the experimental rig and the CFD software.

It was also evident that the production of riblets with WEDM provides tight control on the manufacturing process of intricate geometries, thus ensuring that nature profiles can be replicated for industrial and transportation purposes.

However, further work is required to fully validate the proposed experimental method and to investigate in more depth the proposed structure, in particular in terms of micro structure orientations in accordance to the fluid flow.

\section{References}

[1] Bechert, D. W. et al. eds. 1985. ON THE DRAG REDUCTION OF THE SHARK SKIN. AIAA Paper.

[2] Bechert, D. W. et al. 1997. Experiments on dragreducing surfaces and their optimization with an adjustable geometry. Journal of Fluid Mechanics 338, pp. $59-87$.

[3] Bechert, D. W. et al. 2000. Experiments with threedimensional riblets as an idealized model of shark skin. Experiments in Fluids 28(5), pp. 403-412.

[4] Koeltzsch, K. et al. 2002. Flow over convergent and divergent wall riblets. Experiments in Fluids 33(2), pp. 346-350.

[5] Bixler, G. D. and Bhushan, B. 2014. Rice- and butterfly-wing effect inspired self-cleaning and low drag micro/nanopatterned surfaces in water, oil, and air flow. Nanoscale 6(1), pp. 76-96.

[6] Dean, B. and Bhushan, B. 2012. The effect of riblets in rectangular duct flow. Applied Surface Science 258(8), pp. 3936-3947.

[7] Jin, Y. and Herwig, H. 2014. Turbulent flow and heat transfer in channels with shark skin surfaces: Entropy generation and its physical significance. International Journal of Heat and Mass Transfer 70, pp. 10-22.

[8] Chen, H. et al. 2014. Flow over bio-inspired 3D herringbone wall riblets. Experiments in Fluids 55(3). [9] Pallav, K. et al. 2014. Comparative assessment of 
the laser induced plasma micromachining and the micro-EDM processes. J. of Manufacturing Science and Engineering, Transactions of the ASME 136(1). 